\title{
Control of a Hovering Quadrotor UAV Subject to Periodic Disturbances
}

\author{
Hammad Zaki ${ }^{1}$ and Mustafa Unel ${ }^{1,2}$
}

\begin{abstract}
Quadrotor is a rotary-wing UAV, which has a simple structure but highly nonlinear dynamics. Controlling a hovering quadrotor subject to external disturbances is a crucial task in many applications. In this paper, periodic disturbances have been tackled and novel disturbance observers (DOB) have been developed to estimate the total disturbance acting on the vehicle. It is especially difficult to reject periodic disturbances in low as well as in high frequency region due to the bandwidth limitations of the low-pass filter utilized in conventional DOB. As the cutoff frequency of the low-pass filter is critical, increased bandwidth reduces the robustness which degrades the disturbance rejection performance in the presence of noise. In addition to the lowpass filter, the new structure also consists of a bank of band-pass filters and a high-pass filter. Since the total disturbance acting on the vehicle is compensated by the proposed DOB, PD controllers with feedforward terms are utilized for stabilizing both position and attitude dynamics. Simulation results show the improved robustness obtained by the proposed method.
\end{abstract}

Keywords-Quadrotor, hover, periodic disturbance, disturbance observer, hierarchical control

\section{INTRODUCTION}

Vertical take off and landing (VTOL) vehicles, especially quadrotors have greatly attracted researchers due to their civilian and military applications such as precision farming [1], city monitoring [2] and surveillance [3]. Hovering control of a quadrotor at a desired altitude in an unconstrained environment is a challenging task due to its highly nonlinear dynamics. Robust hovering control of a quadrotor in an unsupervised manner against the external disturbances has gained great interest in the unmanned air vehicle community and many new solutions have been proposed for various problems inherent to the exploitation of its dynamics. Bouabdullah et al. [4] utilized a linear model of a quadrotor and results for PID controller were compared with LQ controller, which showed stability issues in the presence of disturbances. Waslander and Wang [5] used Dryden wind to model wind velocity experienced by the quadrotor and estimated disturbance was used to improve the positioning accuracy. Robust control of a rotary-wing UAVs using a hierarchical structure were considered [6] [9]. It consists of two parts: upper level control is used for the positional dynamics which generates virtual controls and lower level control is used to for the attitude dynamics which provides tracking according to desired angles computed from virtual controls. Desired or reference angles were obtained through analytical formulas. More recently adaptive nonlinear hierarchical control of a tilt-wing quadrotor was developed in

\footnotetext{
${ }^{1}$ Faculty of Engineering and Natural Sciences, Sabanci University, Istanbul 34956, Turkey.

${ }^{2}$ Integrated Manufacturing Technologies Research and Application Center, Sabanci University, Tuzla, Istanbul, Turkey. $\{$ hammadzaki, munel\}@sabanciuniv.edu.
}

[10] and [11]. Vision based approaches have also been utilized to improve the hovering and positioning performances of UAVs [12]. Bin et al. [13] exploited the optical flow technique for hovering control of a nano-quadrotor where it is used in a PD controller to obtain position and velocity feedback signals.

Periodic disturbances in a high frequency region such as harmonics are one of the main serious issues in the motion control. In many industrial applications, conventional disturbance observer (DOB) is used to reject the disturbances due to its simple structure [14] - [15]. Estimated signal which includes uncertainties and external disturbances is fedback as a compensation signal to cancel the disturbance. Disturbances can be estimated if they stay within the bandwidth of the lowpass filter of DOB. In conventional DOB, bandwidth needs to be large enough to suppress all the disturbances within the frequency range. As the cutoff frequency of the lowpass filter ( $\mathrm{Q}$ filter) is very sensitive, periodic disturbance suppression is difficult to achieve with the conventional DOB structure due to noise and robustness constraints [16]. Infinite order disturbance observer (IFDOB) has been proposed for high frequency periodic disturbance rejection in [17], but it is difficult to suppress the low frequency disturbances with this structure. In response to IFDOB, Enhanced Infinite order disturbance observer (EIFDOB) has been presented recently to remove the disturbances in low as well as in the high frequency region [18].

Acceleration feedback control has gained great interest in the control community due to fast response and disturbance rejection performance with more robustness. Jeong et al.[19] proposed an acceleration based disturbance observer (AbDOB) for the attitude control performance of a quadrotor where angular acceleration signals were estimated through the differentiation of the gyro readings. Then the disturbance was estimated by the difference between the nominal control input torque and the estimated control input torque computed from angular acceleration signals. To counteract the bandwidth problem of a disturbance observer, Katsura et al.[20] presented a position acceleration integrated disturbance observer (PAIDO) where an acceleration sensor is implemented to improve the control performance. Recently, novel acceleration feedback control method for robust hovering was presented in [21] where AbDOB was designed for positional dynamics and a nested position, velocity and inner acceleration feedback control structure was developed for attitude dynamics. Hybrid structure was presented in [22] for the quadrotor manipulation system which consists of the acceleration/velocity based disturbance observer in the inner loop. Total disturbances were estimated through linear accelerations and angular velocity measurements and canceled by the DOB in the inner loop. Velocity based disturbance observer (VbDOB) has also been utilized in [7] and [8], especially in the attitude dynamics of 
a UAV due to lack of availability of reliable angular accelerations. Total disturbances which include external disturbances, nonlinear terms and parametric uncertainties are taken into account. As the utilization of the disturbance observer results in linear dynamics, linear model with nominal parameters was considered and only PID type simple controllers were designed for position and attitude control.

In this work, acceleration/velocity based disturbance observers are developed and utilized in a hierarchical control structure to hover a quadrotor type UAV. Acceleration based disturbance observer (AbDOB) is designed for the positional dynamics and velocity based disturbance observer (VbDOB) is used for the control of the attitude dynamics. The proposed new structure of a DOB consists of a high-pass filter (HPF), low-pass filter (LPF) and a bank of band-pass filters (BPF). Difference of two first order low-pass filters with different cutoff frequencies has been used to obtain a band-pass filter. An additional low-pass filter has been added at the end of a bank of band-pass filters in order to remove the phase shift produced due to the combination of band-pass filters. Since the total disturbance is estimated by the proposed DOB and compensated in the control, simple PD controllers with feedforward compesantion are utilized in the hierarchical control system. The proposed control method is verified by numerical simulations performed on a high fidelity model where measurement noise and parametric uncertainties have also been taken into account. Results show the effectiveness of the proposed method in terms of increased robustness.

This paper is organized as follows: Section II presents the quadrotor dynamics. Section III explains the conventional DOB and develops the proposed DOB. Section IV details the controller design. Section V provide the simulation results and related discussions. Finally Section VI concludes the paper with some remarks and indicate possible future directions.

\section{QUADROTOR DYNAMICS}

Quadrotor consists of a cross structure with four propellers connected to the motors at each edge. The crossed configuration shows robustness even the mechanically linked motors are heavier than the frame[23]. Two frames of references are used

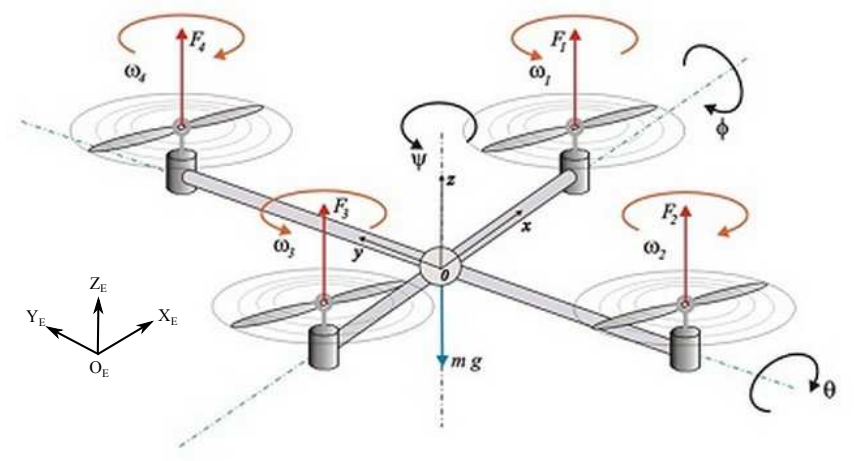

Fig. 1: Quadrotor dynamics (Adapted from [24])

to describe the motion of a quadrotor, one of which is fixed and called inertial frame and the other one which is moving, called body frame. The quadrotor positional dynamics is expressed in the world frame $\left(X_{E}, Y_{E}, Z_{E}\right)$ and attitude dynamics is expressed in the body frame. Considering the quadrotor as a rigid body, its dynamics can be written as

$$
M \dot{\rho}+C(\rho) \rho=G+O(\rho) \omega+E(\varrho) \omega^{2}+D(\rho, \varrho)
$$

where $\rho$ defines the linear velocities in world frame and angular velocities in body frame as

$$
\rho=[\dot{X}, \dot{Y}, \dot{Z}, p, q, r]^{T}
$$

Position and attitude angles are

$$
\varrho=[X, Y, Z, \phi, \theta, \psi]^{T}
$$

Left hand side of (1) consists of the mass-inertia matrix $M$ and Coriolis terms $C$ which are given as

$$
M=\left[\begin{array}{cc}
m\left[I_{3 x 3}\right] & {\left[0_{3 x 3}\right]} \\
{\left[0_{3 x 3}\right]} & \operatorname{diag}\left(I_{x x}, I_{y y}, I_{z z}\right)
\end{array}\right]
$$

where $m$ is the mass, $I_{x x}, I_{y y}$ and $I_{z z}$ are the moment of inertia of the quadrotor about $x, y$ and $z$ axis respectively.

$$
C(\rho)=\left[\begin{array}{cccc}
{\left[0_{3 x 3}\right]} & \multicolumn{3}{c}{\left[0_{3 x 3}\right]} \\
& 0 & I_{z z} r & -I_{y y} q \\
{\left[0_{3 x 3}\right]} & -I_{z z} r & 0 & I_{x x} p \\
& I_{y y} q & -I_{x x} p & 0
\end{array}\right]
$$

Right hand side of (1) consists of gravity term $G$, gyroscopic term $O$ and moment matrix $E$ which are given as

$$
\begin{gathered}
G=[0,0,0,-m g, 0,0,0]^{T} \\
O(\rho) \omega=\left[\begin{array}{c}
{\left[0_{3 x 1}\right]} \\
-J_{T} q \Omega \\
J_{T} p \Omega \\
0
\end{array}\right]
\end{gathered}
$$

where $J_{T}$ is the total moment of inertia around the propeller axis and $\Omega$ is given as

$$
\Omega=\omega_{1}-\omega_{2}-\omega_{3}+\omega_{4}
$$

where $\omega_{1}, \omega_{2}, \omega_{3}$ and $\omega_{4}$ are rotor speeds as shown in Fig1.

$$
E(\varrho) \omega^{2}=\left[\begin{array}{c}
\left(c_{\phi} s_{\theta} c_{\psi}+s_{\phi} s_{\psi}\right) U_{1} \\
\left(c_{\phi} s_{\theta} s_{\psi}-s_{\phi} s_{\psi}\right) U_{1} \\
\left(c_{\phi} c_{\theta}\right) U_{1} \\
U_{2} \\
U_{3} \\
U_{4}
\end{array}\right]
$$

where

$$
\begin{aligned}
& U_{1}=b\left(\omega_{1}^{2}+\omega_{2}^{2}+\omega_{3}^{2}+\omega_{4}^{2}\right) \\
& U_{2}=l b\left(-\omega_{2}^{2}+\omega_{4}^{2}\right) \\
& U_{3}=l b\left(-\omega_{1}^{2}+\omega_{3}^{2}\right) \\
& U_{4}=d\left(-\omega_{1}^{2}+\omega_{2}^{2}-\omega_{3}^{2}+\omega_{4}^{2}\right)
\end{aligned}
$$

where $l, b$ and $d$ are length of rotor arm, thrust factor and drag factor respectively. The following transformation matrix is used to relate angular velocities $(\eta)$ of the quadrotor in body frame to the Euler rates $(\Omega)$ in world frame for attitude control. 


$$
\begin{gathered}
E(\Theta)=\left[\begin{array}{ccc}
1 & s_{\phi} \cdot t_{\theta} & c_{\phi} \cdot t_{\theta} \\
0 & c_{\phi} & -s_{\phi} \\
0 & \frac{s_{\phi}}{c_{\theta}} & \frac{c_{\phi}}{c_{\theta}}
\end{array}\right] \\
\eta=E(\Theta) \Gamma
\end{gathered}
$$

where $\Theta=[\phi, \theta, \psi]^{T}, \Gamma=[\dot{\phi}, \dot{\theta}, \dot{\psi}]^{T}$ and $\eta=[p, q, r]^{T}$. $D(\rho, \varrho)$ in (1) is the periodic disturbances acting on the the quadrotor.

$$
D(\rho, \varrho)=\left[F_{X}, F_{Y}, F_{Z}, \tau_{\phi}, \tau_{\theta}, \tau_{\psi}\right]^{T}
$$

\section{Dob Structures For Periodic Disturbances REJECTION}

The block diagram of the conventional DOB has been shown in Fig. 2, which consists of a simple low-pass filter ( $Q$ filter). $D$ is the added disturbances and $\hat{D}$ is the estimated disturbances. $G_{n}^{-1}(s)$ is the inverse of the nominal plant. $\xi(s)$ represents the sensor noise.

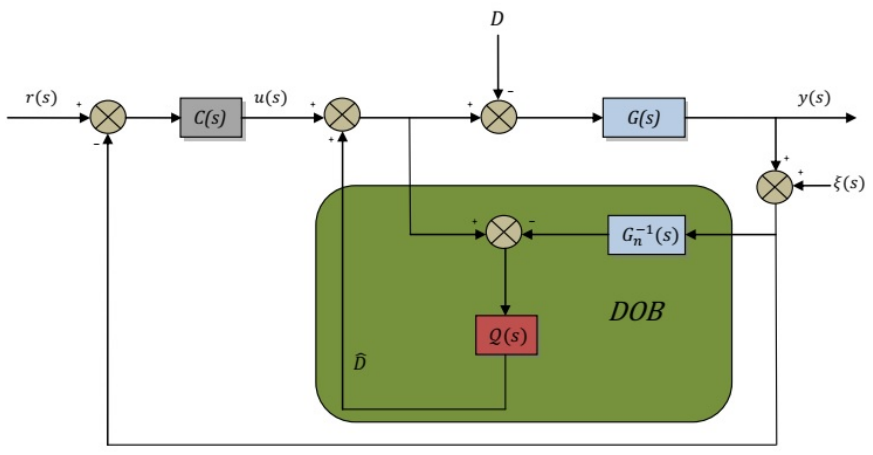

Fig. 2: Conventional DOB based controller

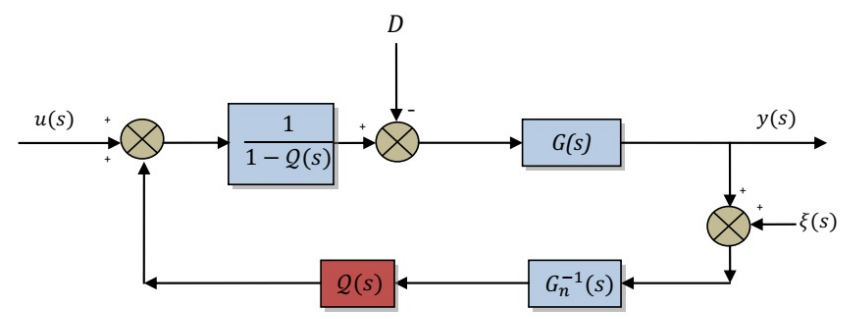

Fig. 3: Realizable DOB loop

From Fig. 2, the transfer function from the inputs $(u, D, \zeta)$ of the DOB loop to the output $(y)$ can be written as

$$
y(s)=G_{D y} D+G_{u y} u(s)+G_{\zeta y} \zeta(s)
$$

where $G_{D y}, G_{u y}$ and $G_{\zeta y}$ are given as

$$
\begin{aligned}
G_{D y} & =\frac{G G_{n}(1-Q)}{Q\left(G-G_{n}\right)+G_{n}} \\
G_{u y} & =\frac{G G_{n}}{Q\left(G-G_{n}\right)+G_{n}} \\
G_{\zeta y} & =\frac{P Q}{Q\left(G-G_{n}\right)+G_{n}}
\end{aligned}
$$

From above transfer functions when $Q \approx 1$, it follows that $G_{D y} \approx 0$ and $G_{u y} \approx G_{n}$. Therefore the total disturbance acting on the system is suppress in the low frequency region and the system is linearized with a nominal transfer function. But at the same time $G_{\zeta y}=1$ and noise will pass unattenuated. When $Q=0$, noise will be blocked, but disturbances will not be rejected and $G_{u y}$ will not be equal to the nominal plant. In order to make the disturbance observer loop realizable, $Q$ cannot be constant. As in motion control, disturbances are considered as low frequency signals, therefore low-pass filter is used for disturbance rejection with carefully adjusted cutoff frequency. Periodic disturbances have generally higher frequency harmonics which are difficult to remove with the help of this structure because of limited bandwidth. Increasing the bandwidth can cause stability issues as it decreases the phase margin of the system.

In order to remove the periodic disturbances in the low and high frequency region, a new structure has been presented in this work to show more robustness. Q filter of the proposed structure is based on the combination of a high-pass filter (HPF), a low-pass filter (LPF) and a bank of band-pass filters (BPF) as shown in Fig 4. Band-pass filters are created through the difference of two first order low-pass filters. Q filter of the modified DOB is given by

$$
Q(s)=\frac{g}{s+g}+\frac{s}{s+g} Q_{1}(s)
$$

where $Q_{1}(s)$ is given by

$$
Q_{1}(s)=\left(\sum_{i=1}^{n} \frac{g_{i+1}}{s+g_{i+1}}-\frac{g_{i}}{s+g_{i}}\right)+\frac{g^{\prime}}{s+g^{\prime}}
$$

In the proposed structure, difference of two LPFs produces some phase lag, therefore an extra low-pass filter has been added to counteract this problem.

\section{CONTROLlER DESIGN UTILIZING ACCELERATION/VELOCITY BASED DistURBANCE OBSERVERS}

Hierarchical control is a useful control technique for UAVs that allows one to design separate controllers for the position and attitude dynamics. The control scheme consists of high level control and low level control. High level controller is used to get necessary desired generalized command signals. Analytical formulas are used to get the desired reference angles $\left(\phi_{d}, \theta_{d}, \psi_{d}\right)$ for low-level attitude control. In order to get robust hovering control, PD controllers along with the estimated disturbance are used as high and low level controllers as shown in closed loop control structure of Fig. 5.

\section{A. Position Control Utilizing Acceleration Based Disturbance Observer}

In order to reject the disturbances acting on the positional dynamics of the quadrotor, acceleration based disturbance observer (AbDOB) has been utilized. AbDOB is used to estimate the total disturbances which include external disturbances, nonlinear terms and parametric uncertainties. The following 


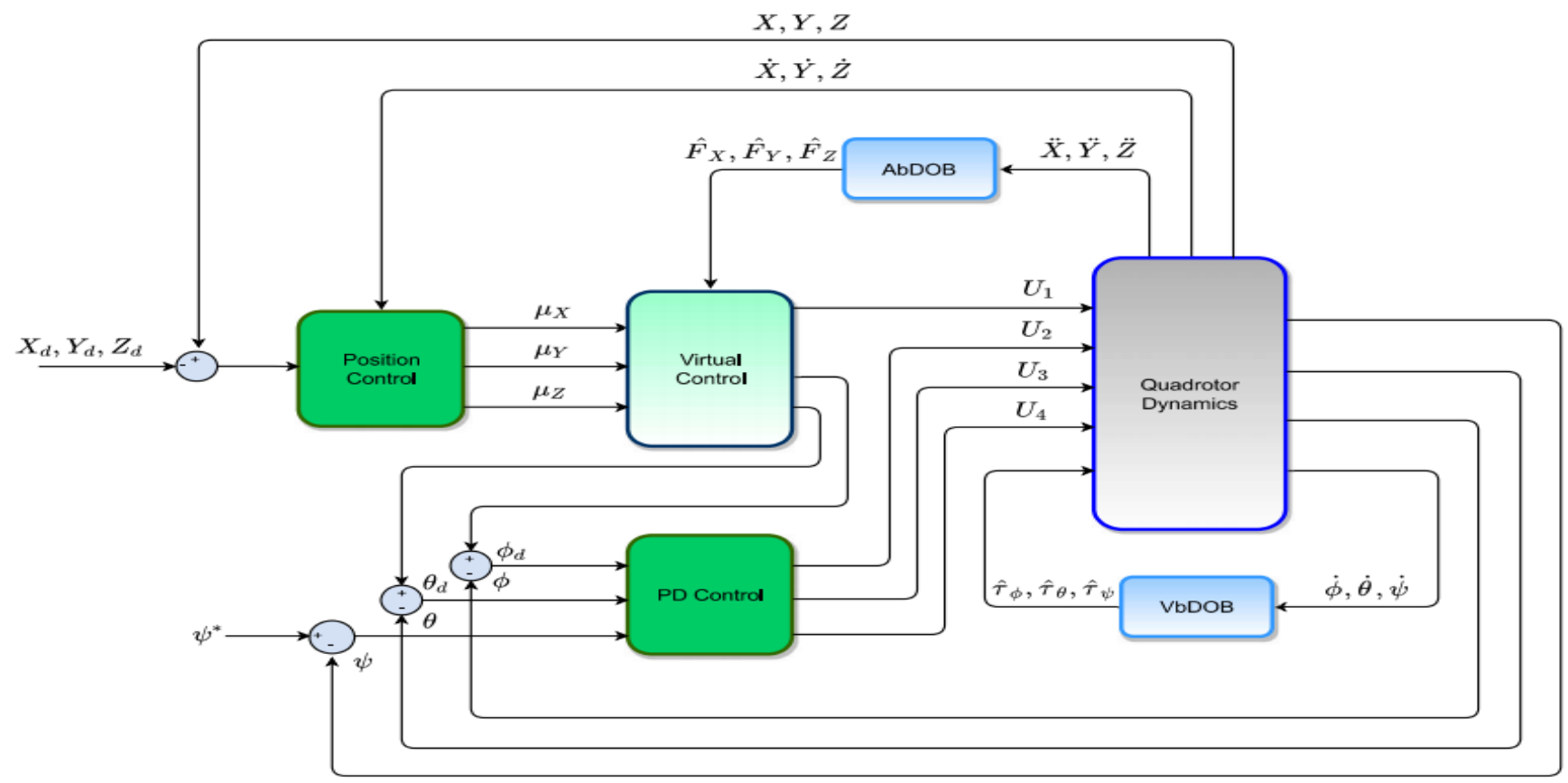

Fig. 5: Closed loop control system

nominal plant has been used in AbDOB.

$$
G_{n}(s)=\left[\begin{array}{ccc}
\frac{1}{m s^{2}} & 0 & 0 \\
0 & \frac{1}{m s^{2}} & 0 \\
0 & 0 & \frac{1}{m s^{2}}
\end{array}\right]
$$

From the positional dynamics of quadrotor in (1)

$$
\begin{aligned}
& \ddot{X}=(\sin \psi \sin \phi+\cos \psi \sin \theta \cos \phi) \frac{U_{1}}{m}+F_{X} \\
& \ddot{Y}=(-\cos \psi \sin \phi+\sin \psi \sin \theta \cos \phi) \frac{U_{1}}{m}+F_{Y} \\
& \ddot{Z}=-g+(\cos \theta \cos \phi) \frac{U_{1}}{m}+F_{Z}
\end{aligned}
$$

where $U_{1}$ is the control input, $F_{X}, F_{Y}$ and $F_{Z}$ are the periodic disturbances. Errors are defined as

$$
e_{X}=X_{d}-X, e_{Y}=Y_{d}-Y, e_{Z}=Z_{d}-Z
$$

Error dynamics can be formulated as

$$
\begin{array}{cl}
\dot{e}_{X}=\dot{X}_{d}-\dot{X} & \Rightarrow \ddot{e}_{X}=\ddot{X}_{d}-\ddot{X} \\
\dot{e}_{Y}=\dot{Y}_{d}-\dot{Y} & \Rightarrow \ddot{e}_{Y}=\ddot{Y}_{d}-\ddot{Y} \\
\dot{e}_{Z}=\dot{Z}_{d}-\dot{Z} & \Rightarrow \ddot{e}_{Z}=\ddot{Z}_{d}-\ddot{Z}
\end{array}
$$

From the positional dynamics, $\ddot{X}$ can be defined as

$$
\ddot{X}=\mu_{X}+F_{X}
$$

Therefore error dynamics in (23) becomes

$$
\ddot{e}_{X}=\ddot{X}_{d}-\mu_{X}-F_{X}
$$

where $\mu_{X}$ is given as

$$
\mu_{X}=\ddot{X}_{d}+K_{d, X} \dot{e}_{X}+K_{p, X} e_{X}-\hat{F}_{X}
$$

PD is used as the feedback controller. $\hat{F}_{X}$ is the estimated disturbance which is used as feedforward term along with the $\ddot{X}_{d}$. Similarly $\mu_{Y}$ and $\mu_{Z}$ are given as

$$
\begin{aligned}
& \mu_{Y}=\ddot{Y}_{d}+K_{d, Y} \dot{e}_{Y}+K_{p, Y} e_{Y}-\hat{F}_{Y} \\
& \mu_{Z}=\ddot{Z}_{d}+K_{d, Z} \dot{e}_{Z}+K_{p, Z} e_{Z}-\hat{F}_{Z}
\end{aligned}
$$

$U_{1}$ can be calculated from $\mu_{x}, \mu_{y}$ and $\mu_{z}$ as [7]:

$$
U_{1}=m \sqrt{\mu_{X}^{2}+\mu_{Y}^{2}+\left(\mu_{Z}+g\right)^{2}}
$$

In order to calculate the desired attitude angles of the aerial vehicle from desired the acceleration vector, yaw angle $(\psi)$ is assumed to be some fixed value $\left(\psi^{*}\right)$. Desired angles are calculated as:

$$
\begin{gathered}
\phi_{d}=-\arcsin \left(\frac{\mu_{Y}}{U_{1}}\right) \\
\theta_{d}=\arcsin \left(\frac{\mu_{X}}{U_{1} \cos \phi_{d}}\right)
\end{gathered}
$$

\section{B. Attitude Control Utilizing Velocity Based Disturbance Ob- server}

Translational motion of the aerial vehicle depends upon the behavior of the roll, pitch and yaw angles, therefore attitude control is an important part for the motion control of the quadrotor. Note that attitude dynamics is fully actuated. With three inputs $\left(U_{2}, U_{3}, U_{4}\right)$ to control three degrees of freedom motion, separate controllers can be designed for each angular motion. Since it is difficult to obtain reliable angular acceleration, velocity based disturbance observer (VbDOB) has been utilized to tackle the disturbances acting on the 


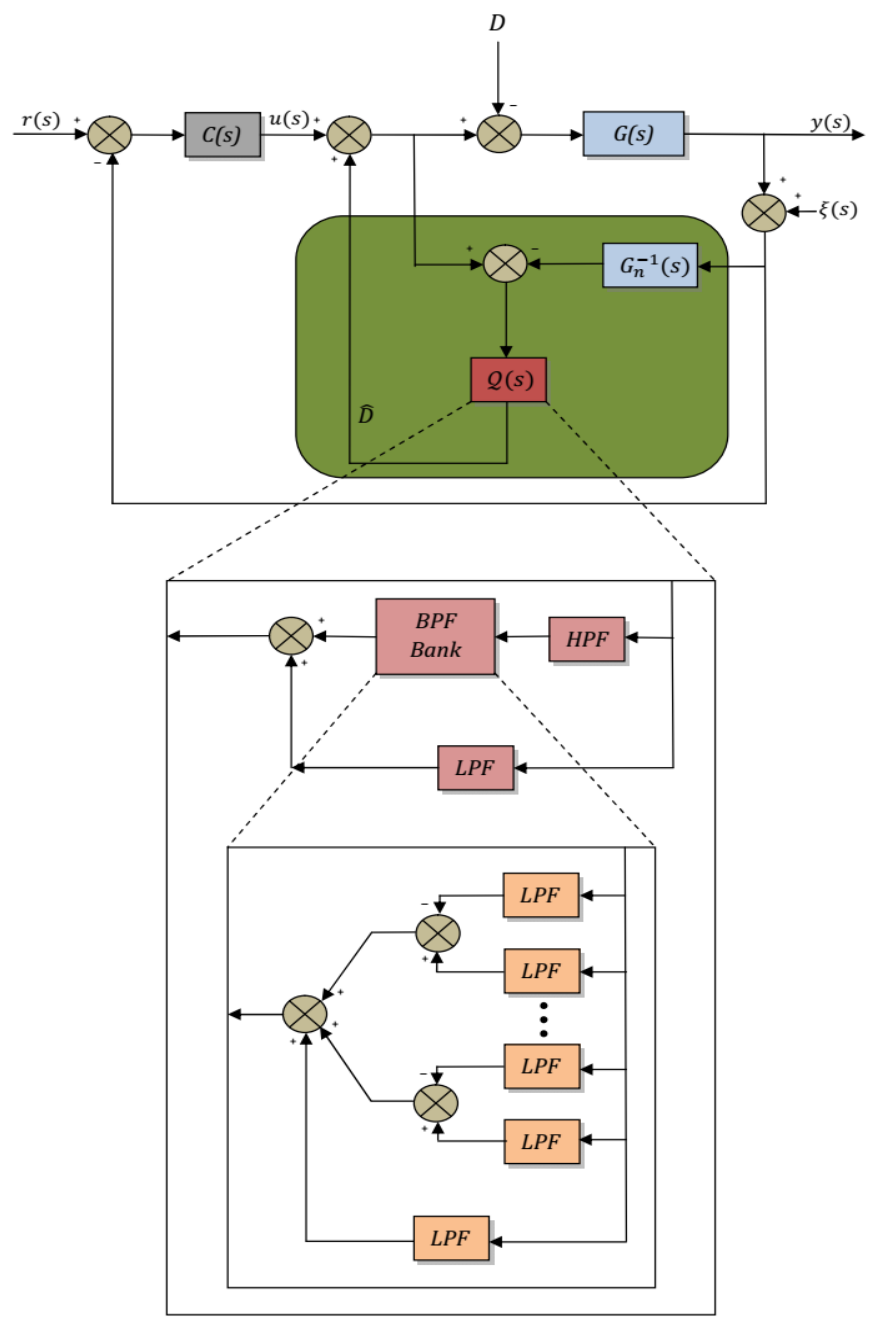

Fig. 4: Proposed DOB structure

attitude dynamics. The following nominal plant has been used in the proposed DOB structure.

$$
G_{n}(s)=\left[\begin{array}{ccc}
\frac{1}{I_{x x} s} & 0 & 0 \\
0 & \frac{1}{I_{y y} s} & 0 \\
0 & 0 & \frac{1}{I_{z z} s}
\end{array}\right]
$$

Errors are defined as

$$
e_{\phi}=\phi_{d}-\phi, e_{\theta}=\theta_{d}-\theta, e_{\psi}=\psi_{d}-\psi
$$

Similarly error dynamics are defined as

$$
\begin{gathered}
\dot{e}_{\phi}=\dot{\phi}_{d}-\dot{\phi} \Rightarrow \ddot{e}_{\phi}=\ddot{\phi}_{d}-\ddot{\phi} \\
\dot{e}_{\theta}=\dot{\theta}_{d}-\dot{\theta} \Rightarrow \ddot{e}_{\theta}=\ddot{\theta}_{d}-\ddot{\theta} \\
\dot{e}_{\psi}=\dot{\psi}_{d}-\dot{\psi} \Rightarrow \ddot{e}_{\psi}=\ddot{\psi}_{d}-\ddot{\psi}
\end{gathered}
$$

In order to develop controllers for attitude control, we first recall the attitude dynamics of the quadrotor.

$$
\dot{p}=\frac{I_{y y}-I_{z z}}{I_{x x}} q r-\frac{J_{T}}{I_{x x}}+\frac{U_{2}}{I_{x x}}+\tau_{\phi}
$$

$$
\begin{gathered}
\dot{q}=\frac{I_{z z}-I_{x x}}{I_{y y}} p r+\frac{J_{T}}{I_{y y}}+\frac{U_{3}}{I_{y y}}+\tau_{\theta} \\
\dot{r}=\frac{I_{x x}-I_{y y}}{I_{z z}} p q+\frac{U_{4}}{I_{z z}}+\tau_{\psi}
\end{gathered}
$$

w0here $\tau_{\phi}, \tau_{\theta}$ and $\tau_{\psi}$ are the disturbances. For controller design, attitude dynamics can be linearized around hover conditions i.e. $\phi, \theta, \psi \approx 0$ and $\dot{\phi}, \dot{\theta}, \dot{\psi} \approx 0$. Angular accelerations in body and world frames will be approximately equal after linearization i.e. $\dot{p} \approx \ddot{\phi}, \dot{q} \approx \ddot{\theta}, \dot{r} \approx \ddot{\psi}$ [7]. Resulting attitude dynamics in (38) can be expressed as

$$
\ddot{\phi}=\frac{U_{2}}{I_{x x}}+\tau_{\phi}
$$

By utilizing the estimated disturbance $\hat{\tau}_{\phi}$, DOB based controller is designed as in [7]. Therefore (41) becomes

$$
\ddot{\phi}=\ddot{\phi}_{d}+K_{d, \phi} \dot{e}_{\phi}+K_{p, \phi} e_{\phi}-\hat{\tau}_{\phi}+\tau_{\phi}
$$

where $U_{2}$ is designed as

$$
U_{2}=I_{x x}\left(\ddot{\phi}_{d}+K_{d, \phi} \dot{e}_{\phi}+K_{p, \phi} e_{\phi}\right)
$$

Similarly, $U_{3}$ and $U_{4}$ are designed by utilizing the corresponding estimated disturbances as

$$
\begin{gathered}
U_{3}=I_{y y}\left(\ddot{\theta}_{d}+K_{d, \theta} \dot{e}_{\theta}+K_{p, \theta} e_{\theta}\right) \\
U_{4}=I_{z z}\left(\ddot{\psi}_{d}+K_{d, \psi} \dot{e}_{\psi}+K_{p, \psi} e_{\psi}\right)
\end{gathered}
$$

where $K_{p, i}>0$ and $K_{d, i}$ are proportional and derivative gains respectively for $i=\phi, \theta, \psi$.

\section{Simulation Results}

In this section disturbance rejection performance of the proposed disturbance observer (DOB) structure is investigated using acceleration based disturbance observer (AbDOB) for positional dynamics and velocity based disturbance observer (VbDOB) for the attitude dynamics. During the simulation, parametric uncertainties and noise are also taken into account to check the robustness of the proposed DOB structure. Following periodic disturbances have been added to the system.

$$
\begin{gathered}
D_{F_{X}, F_{Y}, F_{Z}}=\sum_{l=1}^{5}(2 \sin (2 \pi l 10 t)) \\
D_{\tau_{\phi}, \tau_{\theta}, \tau_{\psi}}=\sin (t)+\sum_{l=1}^{5}(2 \sin (2 \pi l 5 t))
\end{gathered}
$$

Plots for periodic disturbances have been shown in Fig. 6 and Fig. 7. Conventional DOB has also been investigated to compare the results with the proposed disturbance observer. For numerical simulations, parameters are given in the Table I.

Hovering performance has been studied in the presence of periodic disturbances and measurement noise. Position tracking performances have been depicted in Fig. 8, Fig. 9 and Fig. 10. Attitude tracking results have been shown in Fig 11, Fig. 12 and Fig. 13. These results showed better performance in both positioning and attitude tracking for the proposed DOB based controller as compared to classical DOB. From the attitude plots it can be deduced that proposed 

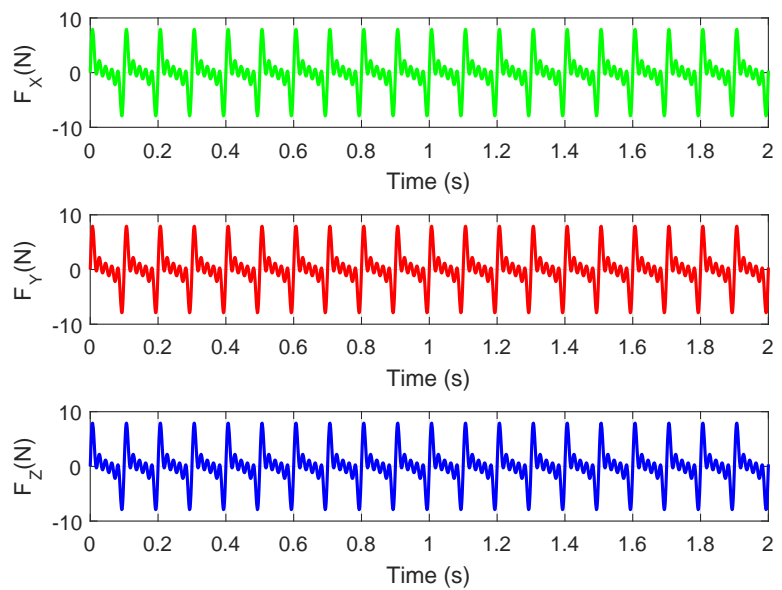

Fig. 6: Periodic disturbances acting on the positional dynamics
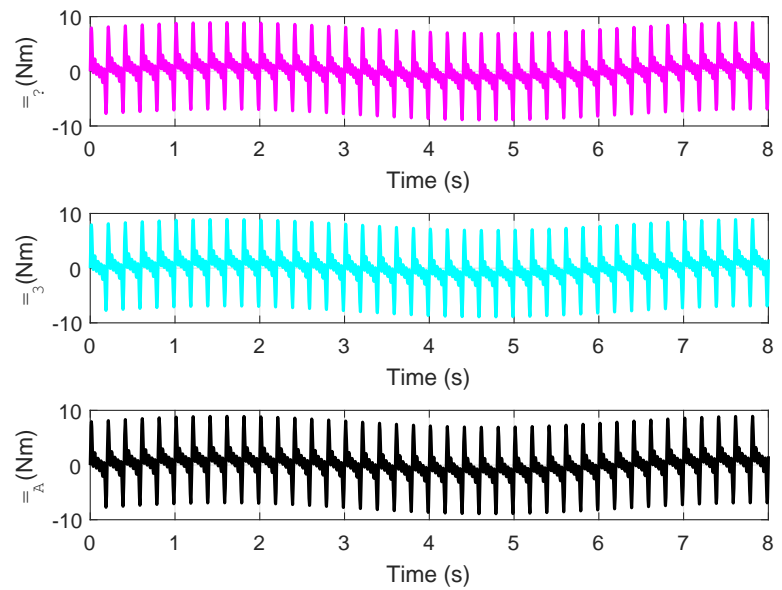

Fig. 7: Periodic disturbances acting on the attitude dynamics

TABLE I: Simulation Parameters

\begin{tabular}{lll}
\hline Symbol & Description & Magnitude \\
\hline$m$ & Mass of vehicle & $1 \mathrm{~kg}$ \\
$I_{x x}$ & Moment of inertia along x axis & $1 \mathrm{e}-01 \mathrm{kgm}^{2}$ \\
$I_{y y}$ & Moment of inertia along y axis & $1 \mathrm{e}-01 \mathrm{kgm}^{2}$ \\
$I_{z z}$ & Moment of inertia along z axis & $15 \mathrm{e}-2 \mathrm{kgm}^{2}$ \\
$g$ & gravity & $9.8 \mathrm{~m} / \mathrm{sec}^{2}$ \\
$l$ & length of the rotor arm & $0.25 \mathrm{~m}$ \\
$K_{p, x}$ & x proportional gain & 3 \\
$K_{d, x}$ & x derivative gain & 2.75 \\
$K_{p, y}$ & y proportional gain & 2.75 \\
$K_{d, y}$ & y derivative gain & 4.25 \\
$K_{p, z}$ & z proportional gain & 1.9 \\
$K_{d, z}$ & z derivative gain & 20 \\
$K_{p, \phi}$ & $\phi$ proportional gain & 2.55 \\
$K_{d, \phi}$ & $\phi$ derivative gain & 1.95 \\
$K_{p, \theta}$ & $\theta$ proportional gain & 7.55 \\
$K_{d, \theta}$ & $\theta$ derivative gain & 8.95 \\
$K_{p, \psi}$ & $\psi$ proportional gain & 10.25 \\
$K_{d, \psi}$ & $\psi$ derivative gain & 3.25 \\
\hline
\end{tabular}

DOB showed smoother results than classical DOB with less peak amplitudes and fluctuations. Position Errors for hovering control at a certain altitude have been depicted in Fig. 14. Table

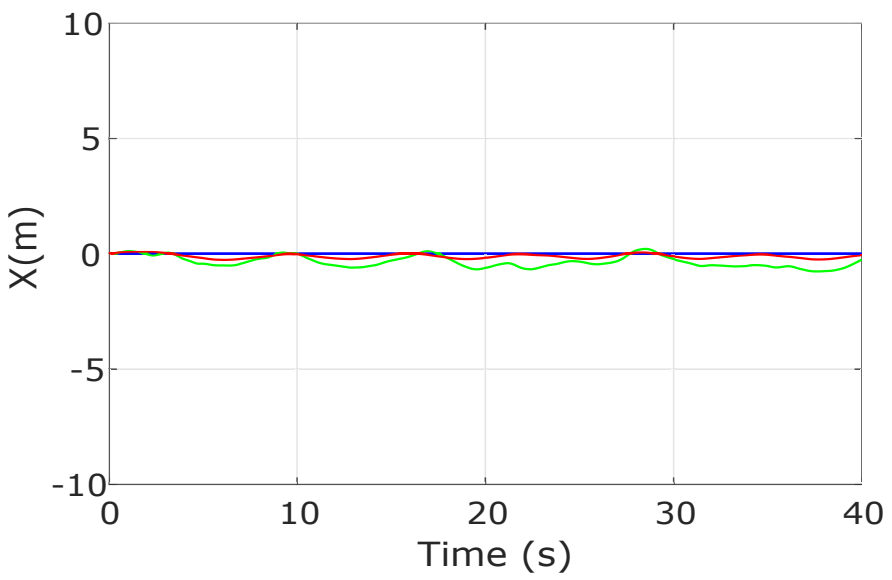

Fig. 8: X Cartesian position of the quadrotor vs Time (Desired=blue, Proposed $\mathrm{DOB}=$ red, $\mathrm{DOB}=$ green)

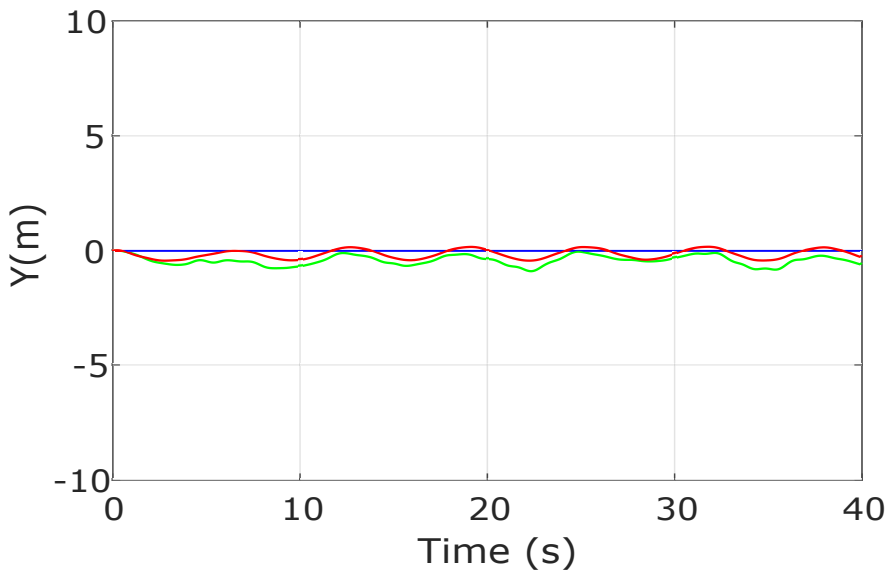

Fig. 9: Y Cartesian position of the quadrotor vs Time (Desired=blue, Proposed $\mathrm{DOB}=$ red, $\mathrm{DOB}=$ green)

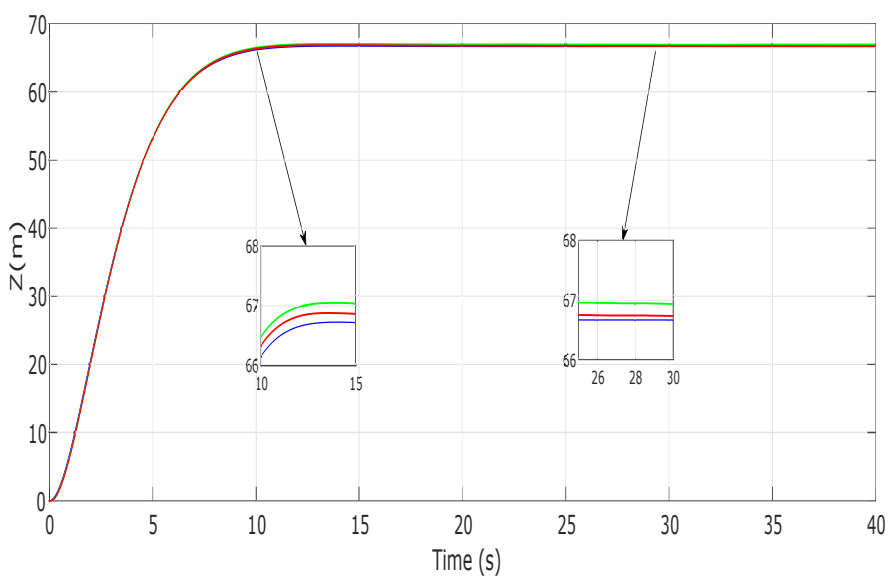

Fig. 10: Z Cartesian position of the quadrotor vs Time (Desired=blue, Proposed $\mathrm{DOB}=\mathrm{red}, \mathrm{DOB}=$ green)

II summarizes the hovering and attitude performance of the quadrotor in terms of root mean square (RMS) and maximum values of the errors. 


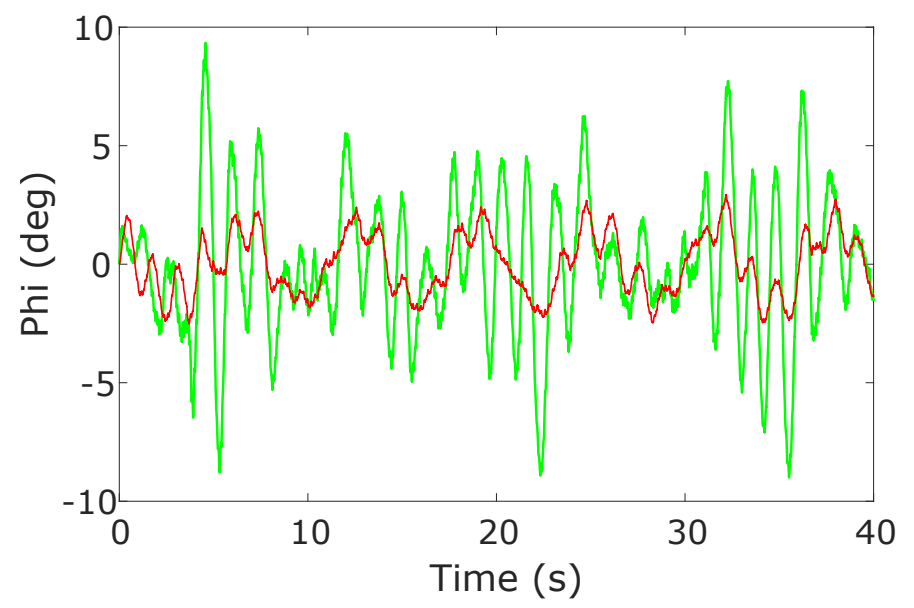

Fig. 11: Roll angle $\phi$ (Proposed DOB=red, DOB=green)

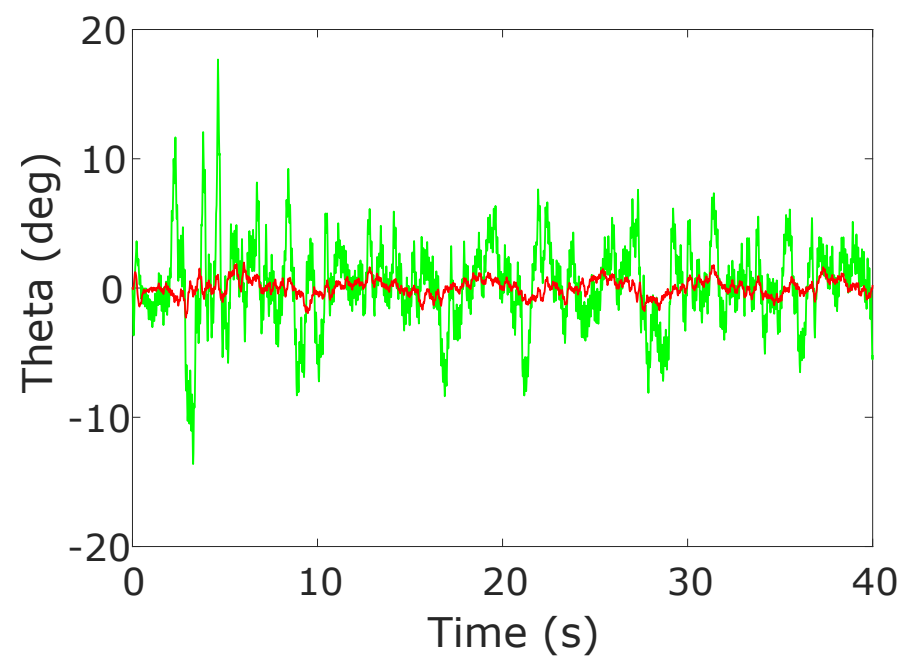

Fig. 12: Pitch angle $\theta$ (Proposed $\mathrm{DOB}=\mathrm{red}, \mathrm{DOB}=$ green)

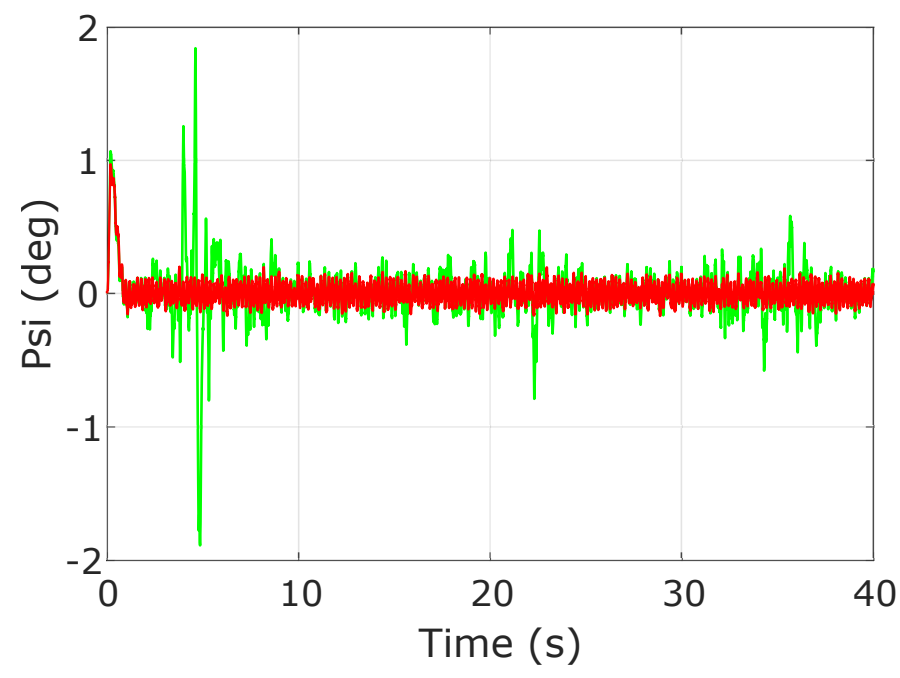

Fig. 13: Yaw angle $\psi$ (Proposed $\mathrm{DOB}=$ red, $\mathrm{DOB}=$ green)

\section{CONClusion}

In this paper, a new structure of DOB based on acceleration and velocity feedback has been presented to suppress the
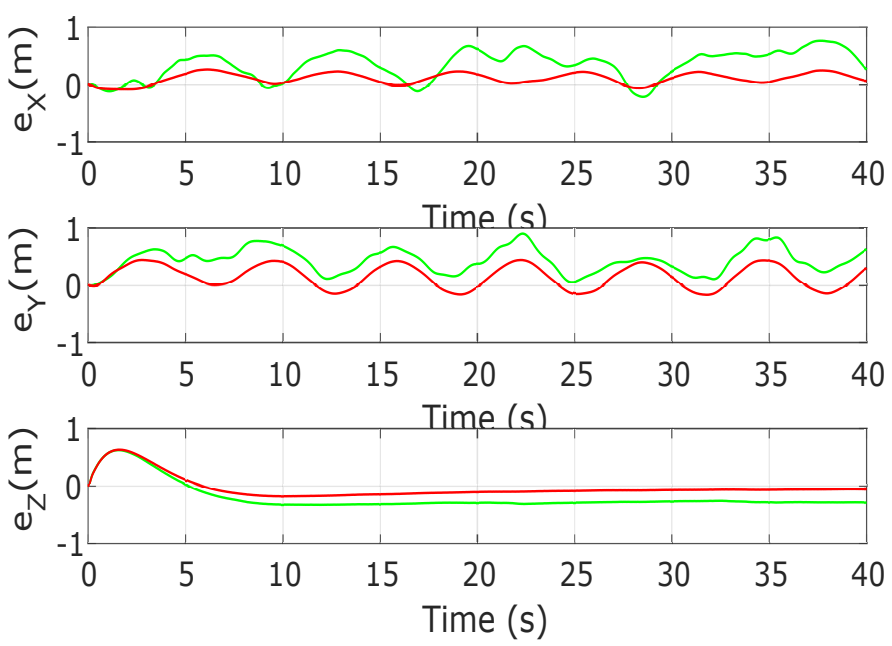

Fig. 14: Position errors (Proposed DOB=red, DOB=green)

TABLE II: Hovering and Attitude Performances

\begin{tabular}{lll}
\hline Criteria & DOB & Proposed DOB \\
\hline$R M S\left(e_{x}\right)(m)$ & 0.4298 & 0.145 \\
$\max \left(\left|e_{x}\right|\right)(m)$ & 0.7683 & 0.2677 \\
$R M S\left(e_{y}\right)(m)$ & 0.4821 & 0.2487 \\
$\max \left(\left|e_{y}\right|\right)(m)$ & 0.9 & 0.4419 \\
$R M S\left(e_{z}\right)(m)$ & 0.3074 & 0.1865 \\
$\max \left(\left|e_{z}\right|\right)(m)$ & 0.6220 & 0.6290 \\
$R M S\left(e_{\phi}\right)($ deg $)$ & 3.05 & 1.293 \\
$\max \left(\left|e_{\phi}\right|\right)(\mathrm{deg})$ & 9.33 & 2.944 \\
$R M S\left(e_{\theta}\right)(\mathrm{deg})$ & 3.21 & 0.64 \\
$\max \left(\left|e_{\theta}\right|\right)(\mathrm{deg})$ & 17.67 & 2.29 \\
$R M S\left(e_{\psi}\right)(\mathrm{deg})$ & 0.22 & 0.1042 \\
$\max \left(\left|e_{\psi}\right|\right)(\mathrm{deg})$ & 1.89 & 0.97 \\
\hline
\end{tabular}

periodic disturbances acting on a quadrotor. Proposed DOB structure consists of a bank of band-pass filters, a low-pass filter and a high-pass filter. Hierarchical control is used as a closed-loop control structure. PD with feedforward compensation is used as a high level controller for the positional dynamics along with acceleration based disturbance observer to obtain desired generalized command signals. Reference angles for the attituede control are calculated analytically from these generalized command signals. PD is also used as a low level controller along with the velocity based disturbance observer for attitude control. Periodic disturbances have been considered to evaluate the efficiency of the proposed DOB in the presence of noise. From simulation results it is clear that the new DOB structure shows better hovering performance than conventional DOB by suppressing the periodic disturbances more effectively.

As a future work, the proposed method will be extended to the 2D and 3D Cartesian trajectory tracking control of a quadrotor. Other structures of DOB for periodic disturbance rejection will also be taken into account for more detailed comparison. The proposed DOB structure will also be tested on a physical system to evaluate the performance in a real environment.

\section{REFERENCES}

[1] Ristorto, G., Mazzetto, F., Guglieri, G., Quagliotti, F.: Monitoring, performances and cost estimation of multirotor unmanned aerial systems in precision farming. In: International Conference on Unmanned Aircraft Systems (ICUAS), Denver, USA, pp. 502-509, June 2015. 
[2] Abdullah, A., Bakar, E.A., Pauzi, M.Z. M.: Monitoring of Traffic Using Unmanned Aerial Vehicle in Malaysia Landscape Perspective. Jurnal Teknologi, Vol. 76, no. 1, pp. 367-372, 2015.

[3] Gupte, S., Mohandas, P.I.T, Conrad, J.M.: A Survey of Quadrotor Unmanned Aerial Vehicles. In: Proceedings of IEEE, Southeastcon, pp. 1-6, March 2012.

[4] Bouabdallah, S., Murrieri, P., Siegwart, R.: Design and Control of an Indoor Micro Quadrotor. In: Proceedings of the 2004 IEEE International Conference on Robotics \& Automation, New Orleans, LA, USA, pp. 43934398, 2004

[5] Waslander, S.L. and Wang, C. Wind disturbance estimation and rejection for quadrotor position control, In AIAA Infotech@ Aerospace Conference and AIAA Unmanned... Unlimited Conference, 2009.

[6] Cetinsoy, E., Dikyar, S., Hancer, C., Oner, K.T., Sirimoglu, E., Unel, M., Aksit, M.F.: Design and Construction of a Novel Quad Tilt-Wing UAV. Mechatronics, Vol. 22, Issue 6, pp 723-745, September 2012.

[7] Hancer, C., Oner, K. T., Sirimoglu, E., Cetinsoy, E., Unel, M.: Robust Position Control of a Tilt-Wing Quadrotor. In: 49th IEEE Conference on Decision and Control, Hilton Atlanta Hotel, Atlanta, GA, USA, December 15-17, 2010.

[8] C. Hancer,C., Oner,K.T., Sirimoglu, E., Cetinsoy, E. and Unel, M.: Robust hovering control of a quad tilt-wing uav, In IECON 2010-36th Annual Conference on IEEE Industrial Electronics Society, pp. 1615-1620, 2010

[9] Zaki H., Unel, M. and Yildiz, Y.: Trajectory Control of a Quadrotor Using a Control Allocation Approach. In: 2017 international conference on unmanned aircraft systems (ICUAS), Miami, FL, USA, 1316 June 2017, pp. 533539. IEEE.

[10] Yildiz, Y., Unel, M., Demirel, A.E.: Adaptive Nonlinear Hierarchical Control of a Quad Tilt-Wing UAV. In: European Control Conference (ECC), Linz, Austria, July 15-17, 2015.

[11] Yildiz, Y., Unel, M., Demirel, A.E.: Nonlinear Hierarchical Control of a Quad-Tilt-Wing UAV: An Adaptive Control Approach. International Journal of Adaptive Control and Signal Processing, DOI:10.1002/acs2759, 2017.

[12] Mathe, K. and Busoniu, L. :Vision and control for UAVs: A survey of general methods and of inexpensive platforms for infrastructure inspection, Sensors, 15(7), 14887-14916, 2015.

[13] Bin, X., Yang, L., Xu, Z., Meihui, C. A. O., Fu, W.: Hovering control of a nano quadrotor unmanned aerial vehicle using optical flow, In Control Conference (CCC), 33rd Chinese, pp. 8259-8264, 2014.

[14] Li, S., Yang, J., Chen, W.H., Chen, X.: Disturbance Observer-Based Control: Methods and Applications. CRC press, Oct. 12, 2017.

[15] Chen, W.H., Yang, J., Guo, L., Li, S.: Disturbance-Observer-Based Control and Related Methodsan Overview. IEEE Transactions on Industrial Electronics, Vol. 63, no. 2, pp. 1083-1095, Feb 2016.

[16] Sariyildiz, E., Ohnishi, K.: Stability and Robustness of Disturbance Observer Based Motion Control System. IEEE Transactions On Industrial Electronics, Vol. 62, No.1, pp. 414-422, 2015.

[17] Muramatsu, H., Katsura, S.: Periodic Disturbances Suppression Based on Infinite-Order Disturbance Observer. In: Proceedings of the IEEJ International workshop on sensing, Actuation, and Motion Control, SAMCON, pp. 2391-2404, 2016

[18] Muramatsu, H., Katsura, S.: Design of an Infinite-Order Disturbance Observer Enhancing Disturbance Suppression Performance. IEEJ Transaction on Industrial Applications, Vol. 6, No 03, pp 192-198, 2017.

[19] Jeong, S. H., Jung, S., Tomizuka, M.: Attitude Control of a QuadRotor System Using an Acceleration Based Disturbance Observer: An Emprical Approach. In: IEEE/ASME Conference on Advanced Intelligent Mechatronics, Kachsiung, Taiwan, pp: 916-921, 2012.

[20] Katsura, S., Irie, K., Ohishi, K.: Wideband Force Control by PositionAcceleration Integrated Disturbance Observer. IEEE Transactions on Industrial Electronics, Vol. 55, no: 4, 2008.

[21] Alcan, G., Unel, M.: Robust Hovering Control of a UAV Using Acceleration Feedback. In: International Conference on Unmanned Aircraft Systems (ICUAS), Miami, FL, USA, 2017.

[22] Kalifa, A., Fanni, M., Namerikawa, T.: Hybrid Acceleration/velocityBased Disturbance Observer for a Quadrotor Manipulation System. In: IEEE conference on Control Applications (CCA), Beunos Aires, Argentina, Sep 19-22, 2016.
[23] Bresciani, T.: Modelling, Identification and Control of a Quadrotor Helicopter, MSc thesis, Department of Automatic Control Lund University, Sweden, Oct 2008.

[24] Hewitt, J.: Drone Athleticism: Astounding New Quadrotor Control, and Beyond, June 14, 2013. Available at: https://www.extremetech.com/extreme/158510-drone-athleticismastounding-new-quadrotor-control-and-beyond. 